\title{
Galerkin Finite-Element Method for the Analysis of the Second Harmonic Generation in Wagon Wheel Fibers
}

\author{
Motahare Sadat Hoseinian, Ali Reza Ahmadi, Maryam Alvanforoush, Ali Asghar Zakerifar, \\ Mohammad Agha Bolorizadeh
}

Physics and Photonics Dept., Graduate University of Advanced Technology, Kerman, IRAN

Corresponding Author Email: mabolori@uk.ac.ir

Received: Jan. 3, 2016, Revised: Jul. 16, 2016, Accepted: Aug. 15, 2016, Available Online: Aug. 8, 2017

DOI: 10.18869 /acadpub.ijop.11.2.113

\begin{abstract}
The nonlinear effects of the second harmonic generation have been investigated for the propagation of light along the axis of fibers of wagon wheel cross sectional shape. Nodal finite element formulation is utilized to obtain discretized Helmholtz equations under appropriate boundary conditions. The hierarchical $p$-version nodal elements are used for meshing the cross section of wagon wheel fiber. The fiber material has been chosen to be $\mathrm{LiTaO}_{3}$ to provide proper second harmonic generation. Propagation of generated second harmonics for two incident field amplitudes are studied in this work.
\end{abstract}

KEYWORDS: Second harmonics, Wagon wheel fiber, Finite element method, $\mathrm{LiTaO}_{3}$.

\section{I.INTRODUCTION}

Nonlinear wave propagation has been investigated by many researchers over the last 30 years. Many interesting phenomena have been observed in the literature due to the theoretical and experimental works reported on nonlinear effects [1-3] such as second harmonic generation in fibers.

In 2001, a full vectorial three-dimensional beam-propagation method (BPM), based on the finite-element formalism, was described for the analysis of second harmonic generation (SHG) in a waveguide [1]. SHG in holey optical fibers was carried out by Monro, et al. [2] in the same year. Holey or microstructured optical fibers (HFs) possess wavelength-scale air holes in the cladding, which lead to a unique range of optical properties. For example, these fibers can endlessly be single mode where the mode area can be tailored over three orders of magnitude.

In 2003, K. Moutzouris et al. [4] report optical second-harmonic generation, SHG, through modal phase matching in GaAs/AlGaAs semiconductor waveguides using femtosecond pulses. They observed both type-I and type-II SHG for input signal wavelength near $1.55 \mu \mathrm{m}$ and obtained practical SHG average powers of up to $10 \mu \mathrm{W}$ with an average input power of $65 \mathrm{~mW}$ for the most efficient type-II interaction.

In 2006, L. Scaccabarozzi et al. [5] observed the $\mathrm{SHG}$ from an $\mathrm{AlGaAs} / \mathrm{Al}_{\mathrm{x}} \mathrm{O}_{\mathrm{y}}$ waveguide which they could enhanced it up to 10 times by forming a waveguide-embedded cavity. In 2011, Rahman et al. [6] designed and analyzed various photonic crystal fibers employing full vectorial FEM approach. They could control the dispersion for supercontinuum generation, the leakage loss and design optimization of SHG.

In 2009, a new generation of fibers consisting of an optical fiber with a suspended micronscale core was made and named wagon wheel fiber [3]. The fiber designs consist of an optical fiber that is partially exposed to the external environment, makes it particularly 
useful for sensing. These fibers allow for strong evanescent field interactions with the surrounding media due to the small core size, providing the potential for real-time and distributed measurements.

In this paper, we will solve full Maxwell's equations to find the second harmonic corrections on the modes of fiber structures known as wagon wheel $[7,8]$ by implementing nodal finite element method. The hierarchical p-version nodal elements of 7 nodes triangular and 9 nodes quadrilateral $[9,10]$ elements are used to mesh the cross section of wagon wheel fiber.

One of the symmetry properties posed by some of the crystals is inversion symmetry. For a material system that is centro-symmetric, the $\chi^{2}$ nonlinear susceptibility vanishes identically. Since the second-order nonlinear interaction is eliminated for all crystals belonging to this class, we use $\mathrm{LiTaO}_{3}$ as wagon wheel fiber material due to its birefringent property.

The eigenvalue problem is solved in $\mathrm{x}-\mathrm{y}$ plane and a set of $\beta_{1}$ s and a set of $\beta_{2}$ s are computed. In the evaluation process, one eigenvalue from each set was selected based on similarity in their corresponding eigenvectors. The set of nonlinear differential equations were solved using Galerkin finite element formulation.

Maxwell's equations are the starting point for any problem in electromagnetism [1]. The Maxwell's equations are simplified as:

$$
\nabla \times \nabla \times \mathbf{E}+\mu_{0} \frac{\partial^{2}}{\partial t^{2}}\left(\varepsilon_{0}[\varepsilon] \mathbf{E}+\varepsilon_{0} \mathbf{P}^{N L}\right)=0(1)
$$

where $\varepsilon_{0}, \mu_{0}$ and $[\varepsilon]$ are the vacuum electric permittivity, vacuum magnetic permeability and the linear relative permittivity tensor of the medium, respectively. The values of the linear relative permittivity tensor elements could be found from references $[11,12] . \mathbf{P}^{N L}$ is the nonlinear polarization vector. If one assumes the space and time dependence of the electric field to be:
$\mathbf{E}(\mathbf{r}, t)=\mathbf{e} e^{j(\omega t-\beta z)}=\left\{\begin{array}{l}e_{u} \\ e_{v} \\ e_{w}\end{array}\right\} e^{j(\omega t-\beta z)}$,

where $\mathbf{e}_{u}, \mathbf{e}_{v}$, and $\mathbf{e}_{w}$ are the three components of the electric field, e. Making use of the method defined in reference [13], the wave Eq. (1) would be simplified as:

$$
\begin{aligned}
\nabla \times & \times \mathbf{E}-\nabla(\nabla \cdot \mathbf{E}) \\
& +\frac{1}{\varepsilon} \nabla\left(\nabla \cdot \mathbf{P}^{N L}\right)-k_{0}^{2}\left(\varepsilon \mathbf{E}+\mathbf{P}^{N L}\right)=0 .
\end{aligned}
$$

The total electric field vector and the nonlinear polarization vectors, respectively, are assumed to be given in the form of a superposition of the input signal $(i=1)$ and the second harmonic $(i=2)$ waves as:

$\mathbf{E}(x, y, z, t)=\frac{1}{2} \sum_{i=1}^{2}\left\{\begin{array}{l}e_{u_{i}} \\ e_{v_{i}} \\ e_{w_{i}}\end{array}\right\} e^{j\left(\omega_{i} t-\beta_{i} z\right)}+c . c$.

and

$\mathbf{P}^{N L}=\frac{1}{2} \sum_{i=1}^{2}\left\{\begin{array}{l}p_{x_{i}} \\ p_{y_{i}} \\ p_{z_{i}}\end{array}\right\} e^{j(-1)^{i} \Delta \beta z} e^{j\left(w_{i} t-\beta_{i} z\right)}+c . c$.

where $p_{x i}, p_{y i}$ and $p_{z i}$ are the components of the second-order nonlinear polarization amplitude, $\mathbf{p}_{i} \cdot \Delta \beta$ is the phase mismatch between the input signal and the generated second harmonic waves defined as $\Delta \beta=\beta_{2}-2 \beta_{1}$. The dependence of the components of the polarization amplitudes, $\mathbf{p}_{i}$, one the electric field components are given in references $[14,17]$ where the optical tensor is defined.

\section{APPLIED FEM}

In this section we devise the finite element method to the present boundary value problem employing a full vectorial case. The steps taken here are: 
1. defining a proper mesh on the cross section of the fiber,

2. selecting interpolation functions,

3. deriving a secular equation by a Galerkin method and

4. solving the secular equation for the $\mathrm{z}$ component of the wave vector, $\boldsymbol{\beta}$.

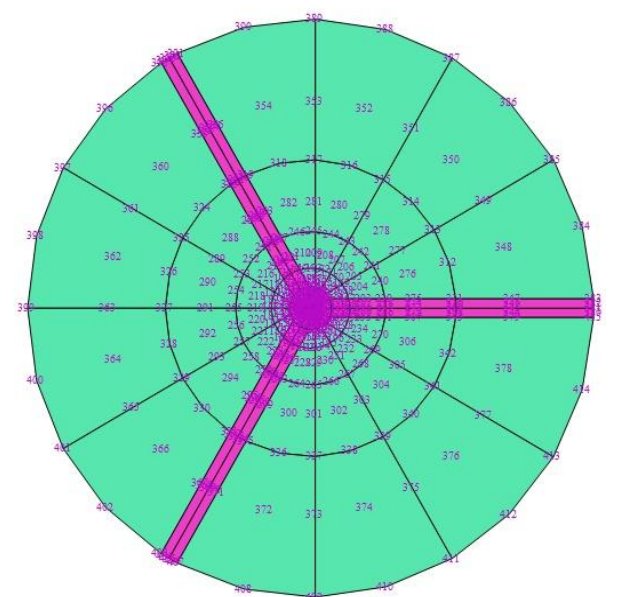

Fig. 1. A typical mesh for wagon wheel fiber's cross sectional view.

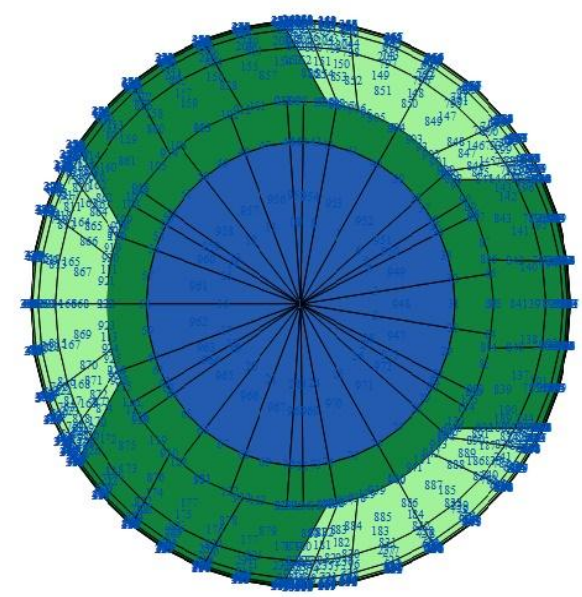

Fig. 2. The cross sectional meshing of fiber shown in Fig. 1 emphasizing the core's mesh.

The objective is to obtain the wave propagation profile using Eq. (3). In this study $\omega$ is assumed as a known parameter and $\beta$ is unknown. Finite element interpolation for each field components can be defined as:

$e_{u}=\sum_{k=1}^{n_{u}} \xi_{k}(x, y) \cdot u_{k}(z)=[\xi]\{u\}$, $e_{v}=\sum_{k=1}^{n_{v}} \eta_{k}(x, y) \cdot v_{k}(z)=[\eta]\{v\}$

and

$e_{w}=\sum_{k=1}^{n_{w}} \psi_{k}(x, y) \cdot w_{k}(z)=[\psi]\{w\}$

where $n_{u}, n_{v}$ and $n_{w}$ are dimensions of interpolation spaces $[\xi],[\eta],[\psi],\{u\},\{v\}$ and $\{w\}$. Therefore, the electric field is written as:

$\mathbf{E}(\mathbf{r}, t)=\boldsymbol{\sigma} \varphi e^{j(\omega t-\beta z)}$

where

$\sigma=\left[\begin{array}{ccc}{[\xi]} & 0 & 0 \\ 0 & {[\eta]} & 0 \\ 0 & 0 & {[\psi]}\end{array}\right]$

and

$\boldsymbol{\varphi}=\left\{\begin{array}{ccc}\{u\} & 0 & 0 \\ 0 & \{v\} & 0 \\ 0 & 0 & \{w\}\end{array}\right\}$

are $\left(n_{u}+n_{v}+n_{w}\right) \times 3$ and $3 \times\left(n_{u}+n_{v}+n_{w}\right)$ dimensional matrices, respectively, where $[\xi]$, $[\eta]$ and $[\psi]$ are vector interpolation functions of two variables $(\mathrm{x}, \mathrm{y})$ and $\{u\},\{v\}$ and $\{w\}$ are vector interpolation functions of single variable (z). Each basis $u, v$ and $w$ is obtained through tensor multiplication of two single dimensional hierarchical interpolation functions. Hierarchical triangular element used here is based on Szabo formulation [9]. A mesh sample for wagon wheel's cross section is shown in Figs. 1 and 2 as defined by the figure captions. Employing the Galerkin method $[18,19]$, we arrive at:

$$
\begin{array}{r}
\left(\nabla \times \mathbf{E}_{i}, \nabla \times \mathbf{E}_{i}^{*}\right)+\left(\nabla \cdot \mathbf{E}_{i}, \nabla \cdot \mathbf{E}_{i}^{*}\right)- \\
k_{0 i}^{2} \varepsilon\left(\mathbf{E}_{i}, \mathbf{E}_{i}^{*}\right)-k_{0 i}^{2}\left(\mathbf{P}^{N L}, \mathbf{E}_{i}^{*}\right)=0
\end{array}
$$

where the inner product $(a, b)$ is defined as the integration over the boundary $\Omega$; i.e. 
$(a, b):=\int_{\Omega} a \cdot b d \Omega$.

Prior to arriving at the propagation equation, one needs to solve for an eigen-problem to obtain the eigen-modes, which yields a series of $\beta_{i}$ s for any input signal of frequency $\omega_{1}$. Rewriting the Eq. (12), the propagation equation included the nonlinear term, $\mathbf{P}^{N L}$, yields [15]:

$$
\begin{aligned}
& {\left[s_{i}\right] \frac{2}{z^{2}}\left\{{ }_{i}\right\} e^{2 j\left(i_{i} i^{z}\right)}+} \\
& {\left[Q_{i}\right] \frac{-}{z}\left\{{ }_{i}\right\} e^{2 j\left(i_{i}{ }^{z} i^{z}\right)} \quad k_{0 i}^{2}\left(p_{i}, E_{i}^{*}\right)=0}
\end{aligned}
$$

where $i$ stands for initial, $i=1$, and generated nonlinear signal, $i=2$, and

$\left[s_{2}\right]=\left[s_{1}\right]=[s]=\left[\begin{array}{ccc}-\xi^{T} \xi & 0 & 0 \\ 0 & -\eta^{T} \eta & 0 \\ 0 & 0 & 0\end{array}\right]$,

$\left[Q_{1}\right]=\left[\begin{array}{ccc}2 j \beta_{1} \xi^{T} \xi & 0 & \xi^{T} \psi_{x} \\ 0 & 2 j \beta_{1} \eta^{T} \eta & \eta^{T} \psi_{x} \\ \psi^{T} \xi_{x} & \psi^{T} \eta_{y} & 0\end{array}\right]$

and

$\left[Q_{2}\right]=\left[\begin{array}{ccc}2 j \beta_{2} \xi^{T} \xi & 0 & \xi^{T} \psi_{x} \\ 0 & 2 j \beta_{2} \eta^{T} \eta & \eta^{T} \psi_{x} \\ \psi^{T} \xi_{x} & \psi^{T} \eta_{y} & 0\end{array}\right]$.

Now, we will generalize the finite element formulation and interpolate in the $\mathrm{z}$ direction to find the solution to Eq. (14), which is the evolution of the input signal and the generation of its second harmonic along the fiber. Therefore, in order to be able to write the Galerkin's form of Eq. 14, let's define:

$\phi=\left\{\begin{array}{l}u \\ v \\ w\end{array}\right\}_{n \times 1}=\left\{\begin{array}{c}\phi_{1} \\ \vdots \\ \phi_{n}\end{array}\right\}$

$$
\begin{aligned}
\phi_{i} & =\left\{\begin{array}{l}
\sum_{j=1}^{p+1} N_{j}(z) u_{i, j} \\
\sum_{j=1}^{p+1} N_{j}(z) v_{i, j} \\
\sum_{j=1}^{p+1} N_{j}(z) w_{i, j}
\end{array}\right\}, \\
M & =\left[\begin{array}{ccc}
M_{u}(z) & 0 & 0 \\
0 & M_{v}(z) & 0 \\
0 & 0 & M_{w}(z)
\end{array}\right]
\end{aligned}
$$

and

$$
\Phi=\left\{\begin{array}{c}
\left\{\left\{u_{i, j}\right\}_{j=1, p+1}\right\}_{i, n_{u}} \\
\left\{\left\{v_{i, j}\right\}_{j=1, p+1}\right\}_{i, n_{v}} \\
\left\{\left\{w_{i, j}\right\}_{j=1, p+1}\right\}_{i, n_{w}}
\end{array}\right\}
$$

where $p$ is the order of approximation along the $z$ direction.

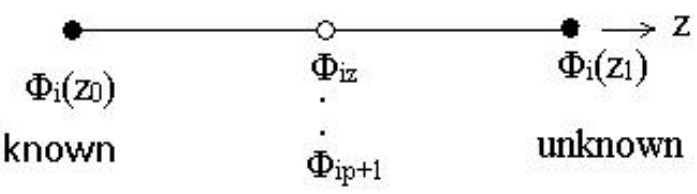

Fig. 3. A sample of element in $\mathrm{z}$ direction.

Therefore, the Galerkin form of Eq. (14) takes the form:

$$
\begin{aligned}
& G(\Phi)=-\left\{\begin{array}{cc}
{\left[\frac{d M}{d z}[s]_{1} \frac{d M}{d z}\right.} & {[0]} \\
{[0]} & \frac{d M}{d z}[s]_{2} \frac{d M}{d z}
\end{array}\right] \\
& +\left[\begin{array}{cc}
M^{T}[Q]_{1} \frac{d M}{d z} & {[0]} \\
{[0]} & {[Q]_{2} \frac{d M}{d z}}
\end{array}\right]+\left[\begin{array}{l}
-M^{T} k_{1}^{2} \alpha \sigma^{T} d L_{1} \\
-M^{T} k_{2}^{2} \gamma \sigma^{T} d L_{2}
\end{array}\right] \\
& \left.\times\left[\begin{array}{cc}
\sigma & 0 \\
0 & \sigma
\end{array}\right]\left[\begin{array}{cc}
M & 0 \\
0 & M
\end{array}\right]\right\}\left\{\begin{array}{l}
\Phi_{1} \\
\Phi_{2}
\end{array}\right\}=\left\{\begin{array}{l}
0 \\
0 \\
0
\end{array}\right\}
\end{aligned}
$$

where the quantities $\Phi_{\mathrm{i}}$ are defined along the $\mathrm{z}$-axis as in Fig 3. 
The nonlinear set of equations given in (22), are solved by applying Newton-Raphson root finding technique, hence,

$\{0\}=G(\Phi)=\left.G\right|_{\left(\Phi=\Phi_{0}\right)}+\left.\delta_{\Phi} G\right|_{\left(\Phi=\Phi_{0}\right)} \Delta \Phi+\ldots$.

Let's define $J\left(\Phi_{0}\right)=\left.\delta_{\Phi} G\right|_{\left(\Phi=\Phi_{0}\right)}$, therefore, we conclude that:

$\left.G\right|_{\left(\Phi=\Phi_{0}\right)}=-J\left(\Phi_{0}\right) \Delta \Phi$

to the first order approximation in $|\Delta \Phi|$. The convergence tolerance for this procedure was set at $|\Delta \Phi|<10^{-6}$.

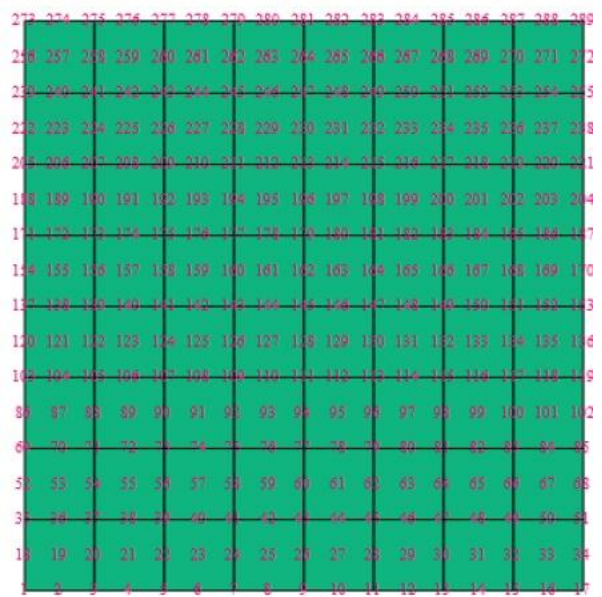

Fig 4. The mesh used to find the modes of a rectangular cross section waveguide in the present FEM method.

\section{III.RESULTS AND DISCUSSION}

The method devised, here, was coded into the SyNA computational tool [20] developed at Kerman Graduate University of Technology. In order to check the validity of the method devised and the computer code developed, the numerical solution for SHG in a typical rectangular waveguide was calculated analytically as well. The analytical solutions to a typical rectangular waveguide of cross section of $L_{\mathrm{x}} L_{\mathrm{y}}$ is a textbook problem and the modes for mode numbers $m$ and $n$ are:

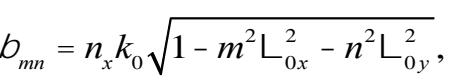

where $n_{x}$ is the refractive index along the $x$ axis for this birefringence material. The quantities $\Lambda_{0 x}$ and $\Lambda_{0 y}$ are defined as $\frac{\lambda_{0}}{2 n_{z} L_{x}}$ and $\frac{\lambda_{0}}{2 n_{x} L_{y}}$, respectively, where $\lambda_{0}$ is the wavelength of the incident signal and $n_{z}$ is the refractive index along the $z$ axis.

Table 1: Analytical and numerical results of $\beta_{1}$ and $\beta_{2}$ in a rectangular waveguide at wavelength $0.866 \mu \mathrm{m}$ for four modes.

\begin{tabular}{|c|c|c|c|c|}
\hline \multirow{2}{*}{$\begin{array}{l}\text { Solution } \\
\text { Mode } \beta \mathrm{s}\end{array}$} & \multicolumn{2}{|c|}{ Analytical } & \multicolumn{2}{c|}{ Numerical } \\
\cline { 3 - 5 } No. & $\beta_{1}$ & $\beta_{2}$ & $\beta_{1}$ & $\beta_{2}$ \\
\hline 5 & 15.55 & 31.11 & 15.55 & 31.11 \\
\hline 8 & 15.54 & 31.08 & 15.54 & 31.07 \\
\hline 23 & 15.48 & 30.96 & 15.47 & 30.94 \\
\hline 38 & 15.32 & 30.65 & 15.37 & 30.74 \\
\hline
\end{tabular}
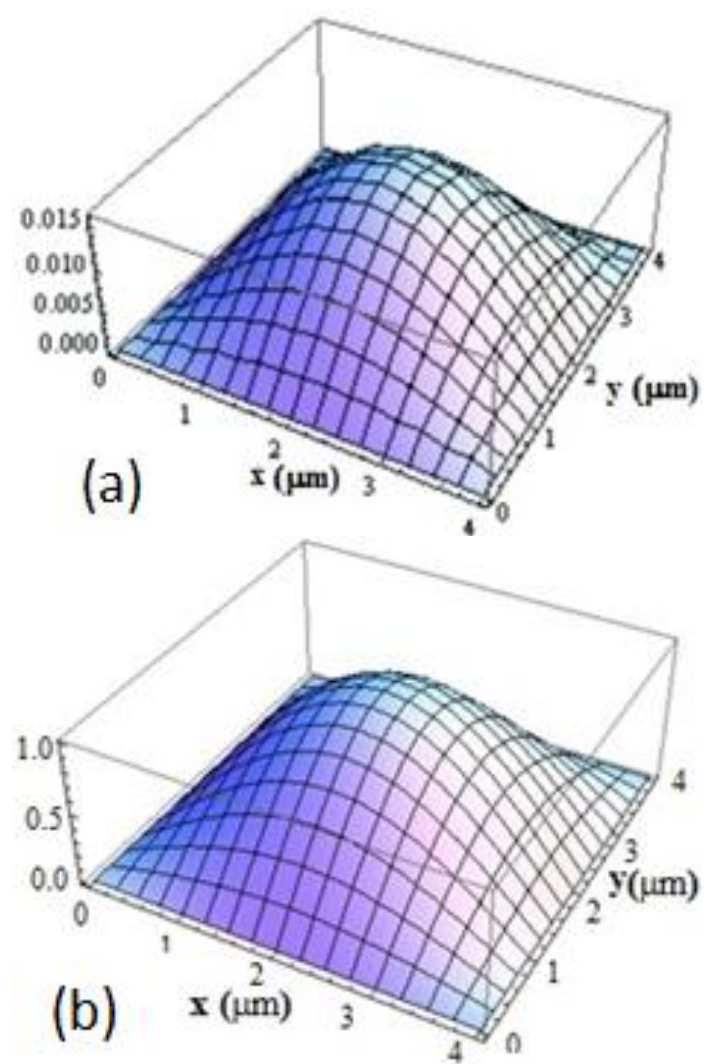

Fig. 5. The electric field distribution for the fundamental mode of the rectangular waveguide at $\beta_{1}=15.55$ found (a) numerically and (b) analytically. Vertical coordinate is the relative Electric Field amplitude. 
The generated mesh for the rectangular waveguide cross section is presented in Fig. 4. The results of $\beta_{1}$ s and $\beta_{2}$ s at 4 different modes are tabulated in Table 1, where the numerical and analytical results agree better than $0.1 \%$. The electric field distributions at the fiber's cross section for the fundamental mode (mode number 5) is plotted in Figs. 5 employing the numerical and analytical calculations, respectively. Comparing the data for $\beta_{1} \mathrm{~s}$ and $\beta_{2} \mathrm{~s}$, shown in Table 1 and the electric field distributions (shown in Figs. 5) approve our consistency check for the model and numerical code developed in this work. Employing the method devised and the computer code developed, we calculated the second harmonic generation in a wagon wheel fiber type and the propagation of a defined input signal in it.

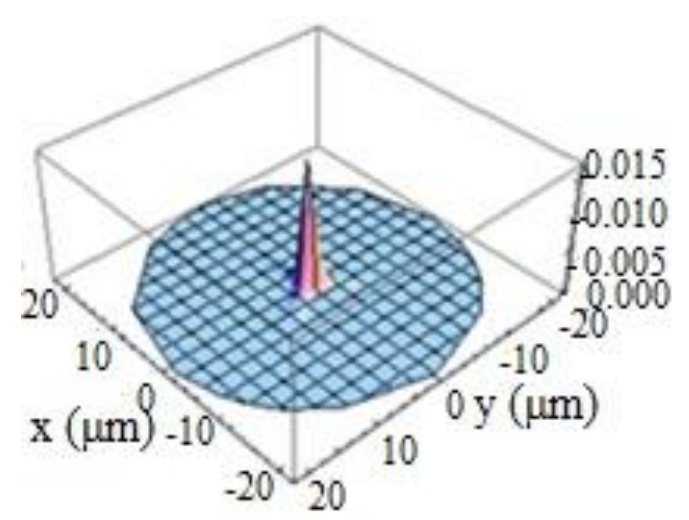

(a)

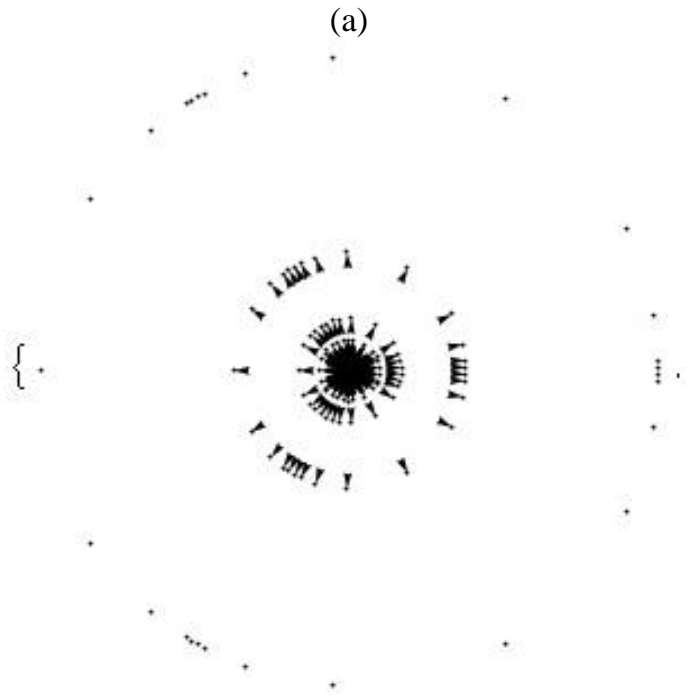

(b)

Fig. 6. Electric field distribution of mode number 1 for SHG $\left(\beta_{2}=32.718\right)$ at the WW fiber's cross section. (a) Three dimensional plot, and (b) planar diagram. Vertical coordinate is the relative amplitude of electric field.
A wagon wheel fiber, whose core is made from $\mathrm{LiTaO}_{3}$, was assumed while the rest of the wagon wheel's material is pure glass in the present study of nonlinear effects of wagon wheel fibers. The core diameter and the outer diameter of the fiber are, respectively, $3.19 \mu \mathrm{m}$ and $43.01 \mu \mathrm{m}$. The wavelength of the input signal, $\lambda_{0}$, is assumed to be equal to $0.866 \mu \mathrm{m}$. The ordinary and the extraordinary refractive indices [11] for $\mathrm{LiTaO}_{3}$ at $\lambda_{0}$ and $\lambda_{0} / 2$ are $n_{\mathrm{o}}\left(\lambda_{0}\right)=2.148, \quad n_{\mathrm{e}}\left(\lambda_{0}\right)=2.152, \quad n_{\mathrm{e}}\left(\lambda_{0} / 2\right)=2.253$, and $n_{0}\left(\lambda_{0} / 2\right)=2.258$. The non-zero nonlinear elements of optical tensor $[21,22]$ for $\mathrm{LiTaO}_{3}$ are $d_{33}=-27(\mathrm{pm} / \mathrm{V}), d_{31}=d_{15}=-4.7(\mathrm{pm} / \mathrm{V})$ and $d_{22}=2.2(\mathrm{pm} / \mathrm{V})$. Field distribution of mode number 1 for SHG $\left(\beta_{2}=32.718\right)$ in the $x-y$ plane is shown in Fig. 6.

In Table 2 , we list the values of $\Delta \beta=\beta_{2}-2 \beta_{1}$ for the first four modes. It indicates that there is a mismatch in phase between the input signal and the second harmonic waves, which increases with increasing mode number. Note that the modes with a number of degeneracies are considered as one mode.

Table 2: The eigenvalues $\beta_{1}$ and $\beta_{2}$ at four mode numbers for a fiber with core and lateral diameters of $3.19 \mu \mathrm{m}$ and $43.01 \mu \mathrm{m}$, respectively.

\begin{tabular}{|l|l|l|l|l|}
\hline Mode No. & 1 & 5 & 7 & 10 \\
\hline$\beta_{1}$ & 15.544 & 15.515 & 15.449 & 15.420 \\
\hline$\beta_{2}$ & 32.718 & 32.667 & 32.655 & 32.655 \\
\hline$\Delta \beta$ & 1.63 & 1.63 & 1.75 & 1.81 \\
\hline
\end{tabular}

Propagation of the input signal, $w_{1}$, along the fiber ( $z$-direction) with $\beta_{1}=15.544$ and generated second harmonic field amplitude, $w_{2}$, along the fiber with $\beta_{2}=32.718$ is studied. An initial signal intensity defined as $\Phi_{0}$ with known values of $u, v$ and $w$ at $z=0$ is used as the initial configuration for the propagation of the electric field in the fiber to start the Newton-Raphson root finding technique. Three cases with initial signal intensities of $1000 \Phi_{0}, 100 \Phi_{0}$ and $10 \Phi_{0}$ are studied here and the propagation of maximum amplitude along the fiber for each case is shown in Fig. 7(a-c) respectively. 
The two curves cited in Fig. 7(a-c) cross at 222 $\mu \mathrm{m}, 261 \mu \mathrm{m}$ and $283 \mu \mathrm{m}$, respectively, showing that nonlinearity effects increase with increasing the input signal intensity as expected.

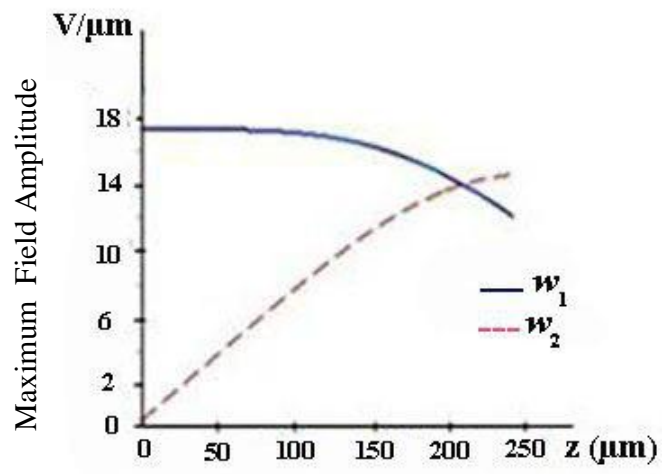

(a)

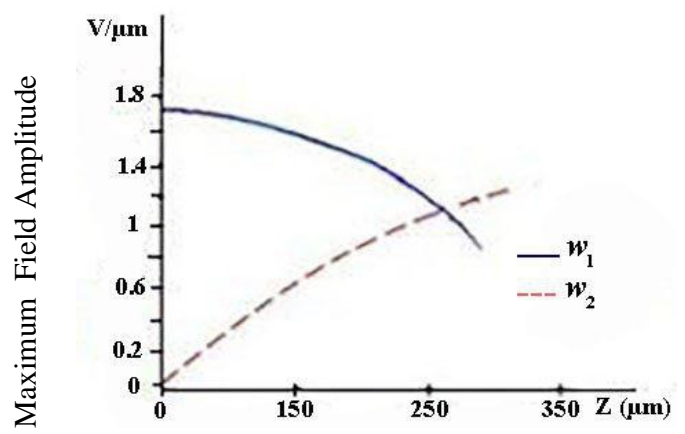

(b)

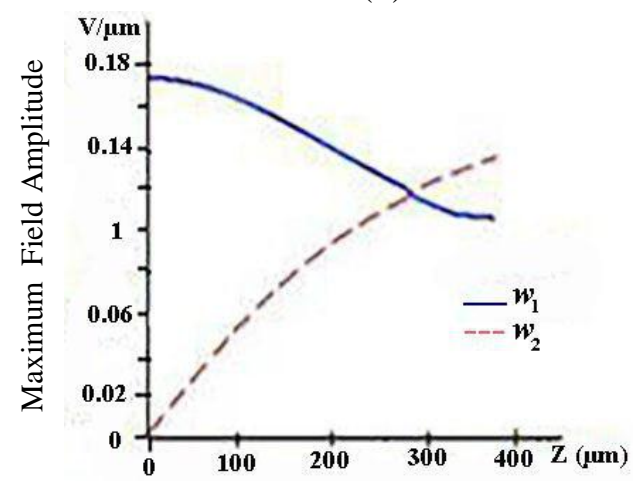

(c)

Fig. 7. The evolution of maximum amplitude along the fiber for the initial signal of (a) $1000 \Phi_{0}$, (b) $100 \Phi_{0}$, and (c) $10 \Phi_{0}$ at $\mathrm{z}=0$, where $w_{1}$ and $w_{2}$ are the field amplitudes of input signal and the generated second harmonic, respectively.

Also the intensity of $w_{2}$ wave, in the three cases examined, is increasing as the input signal advances along the $\mathrm{z}$-axes, which signifies that the second harmonic generation along the fiber is enhancing. Furthermore, the intensity of the input signal, $w_{1}$, is decreasing. Therefore, the input field is dampening along the fiber.

\section{IV.CONCLUSION}

The second harmonic generation along a wagon wheel type fiber of core made from SHG active material $\mathrm{LiTaO}_{3}$ was investigated for three intense pulses at amplitudes of multiples of 10. Making use of the Galerkin finite element formalism, the set of nonlinear differential equations were solved. The eigenvalue problem solved in the $x-y$ plane and a set of $\beta_{1} \mathrm{~s}$, eigen-mode of the initial signal, and a set of $\beta_{2} \mathrm{~s}$, eigen-mode of SHG signal, are calculated as listed in Table 2. In the evaluation process, one eigenvalue from each set was selected based on similarity in their corresponding eigenvectors.

Input signal, $w_{1}$, field amplitude in $\mathrm{z}$ direction with $\beta_{1}=15.544$ and second harmonic $w_{2}$ field amplitude in $\mathrm{z}$ direction with $\beta_{2}=32.718$ as a function of distance was calculated that is the evidence of SHG enhancement in wagon wheel fiber having a core made from $\mathrm{LiTaO}_{3}$. The second harmonic generated in the core enhanced more efficiently as the input signal amplitude was increased and reaching a higher amplitude as compared with the input signal at a closer distance in the fiber.

As this is the first such work for the second harmonic generation and there are no theoretical or experimental results, in the literature, we could not compare the results with any other work. However, in order to test the accuracy of the FEM code devised, the generated second harmonic in a waveguide with a square cross section was obtained and compared with the analytical results which are textbook level problem and it was easily obtained. The agreement shows that the method properly describes the system devised. We recommend more work on the topic of SHG from wagon wheel type fibers. This is of value in the medical and industrical applications. More importantly, SHG is more 
intense when compared with the third harmonic generation, THG.

\section{REFERENCES}

[1] T. Yasui and M. Koshiba, "Three-dimensional vector beam-propagation method for second harmonic generation analysis," J. Lightwave Technol. Vol. 19, pp. 780-785, 2001.

[2] T.M. Monro, V. Pruneri, N.G.R. Broderick, D. Faccio, P.G. Kazansky, and D.J. Richardson, "Broad-Band Second-harmonic generation in holey optical fibers," IEEE Photon. Technol. Lett. Vol. 13, pp. 981-983, 2001.

[3] S.C. Warren-Smith, H. Ebendorff-Heidepriem, T.C. Foo, R. Moore, C. Davis, and T.M. Monro, "Exposed-core microstructured optical fibers for real-time fluorescence sensing," Opt. Exp. Vol. 17, pp. 18533-18542, 2009.

[4] K. Moutazouris, S. Venugopal Rao, M. Ebrahimzadeh, A. De Rossi, M. Calligaro, V. Ortiz, V. Ortiz, and V. Berger, "Second harmonic generation through optimized modal phase matching in semiconductor waveguide," Appl. Phys. Lett. Vol. 83, pp. 620-623, 2003.

[5] L. Scaccabarozzi, M.M. Fejer, Y. Huo, S. Fan, $\mathrm{X}$. $\mathrm{Yu}$, and J.S. Harris, "Enhanced secondharmonic generation in $\mathrm{AlGaAs} / \mathrm{Al}_{\mathrm{x}} \mathrm{O}_{\mathrm{y}}$ tightly confining waveguides and resonant cavities," Opt. Lett. Vol. 31, pp. 3626-3628, 2006.

[6] B.M.A. Rahman, N. Kejalakshmy, A. Agrawal, M. Uthman, I.N.M. Wijeratne, and K.T.V. Grattan, "Finite element characterization of photonic crystal fibers", International Microwave \& Optoelectronics Conference (IMOC), Brazil, pp. 660-664, 2011.

[7] Y. Zhu, R.T. Bise, J. Kanka, P. Peterka and H. Du, "Fabrication and characterization of solidcore photonic crystal fiber for strong evanescent field overlap," Opt. Commun. Vol. 281, pp. 55-60, 2008.

[8] Y. Zhu, H. Du, and R. Bise, "Design of solidcore microstructured optical fiber with steering-wheel air cladding for optimal evanescent- field sensing," Opt. Exp. Vol. 14, pp. 3541-3546, 2006.

[9] S. Adjerid, M. Ai, and J.E. Flaherty, "Hierarchical finite element bases for triangular and tetrahedral elements," Computer Meth. Appl. Mech. Eng. Vol. 190, pp. 2925-2941, 2001.

[10]A. Konrad, Triangular finite elements for vector fields in electro-magnetics, $\mathrm{PhD}$ Thesis, Department of Electrical Engineering, McGill University, 1974.

[11] M. Koshiba, Optical wavegiuide theory by the finite element method, Tokyo: KTK Scientific Publishers, Ch. 2, 1992.

[12] G.P. Agrawal, Nonlinear fiber optics, New York: Academic Press Inc., Ch. 6, 1995.

[13]B.-N. Jiang, L.A. Povineli, and J. Wu, "The origin of spurious solutions in computational electromagnetics," J. Comp. Phys. vol. 125, pp. 1597-1630, 1996.

[14]R.W. Boyd, Nonlinear optics, Boston: Academic Press, Ch. 2, 2003.

[15]P. Gunter, Nonlinear optical effects and material, New York: Springer, Ch. 2, 1999.

[16]P. Banerjee, Nonlinear optics, New York: Marcel Dekker Inc., Ch. 3, 2004.

[17]P. Mandel, Nonlinear optics, Germany: Wiley-VCH Verlag GmbH, Ch. 3, 2010.

[18]N. Reddy, An introduction to the finite element method, New York: McGraw-Hill, Ch. 4, 1984.

[19]J. Jin, The finite element method in Electromagnetics, New York: Wiley-IEEE Press, Ch. 4, 2002.

[20]Z. Dehghanifard, A.R. Ahmadi, A.R. Ganjovi, and M.A. Bolorizadeh, "Space-Time Coupled Finite Element Simulation of PECVD Reactor", Int. J. Appl. Comput. Math. Vol. 2, pp. 1-11 (2015).

[21]F.C. Allard, Fiber optics handbook, New York: McGraw-Hill, Ch.5, 1990.

[22] V.G. Dmitriev, G. G. Gurzadayan, D.N. Nikogosyan, Handbook of nonlinear optics crystals, New York: Springer-Verlag, Ch. 5, 1991. 


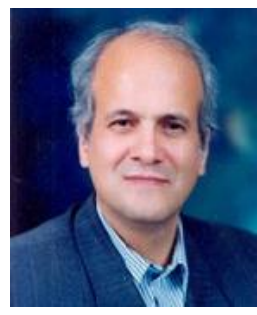

Mohammad Agha Bolorizadeh earned his B.Sc. in Physics from Shiraz University, Shiraz, Iran. Later, he earned his M.Sc. and $\mathrm{PhD}$ in atomic and molecular physics from University of Nebraska-Lincoln, Lincoln, Nebraska in 1979 and 1984, respectively.He started his career at the Shahid Bahonar University of Kerman, Kerman, Iran as assistant professor in 1984. He later (in 2009) joined the Graduate University of Advanced Technology (GUAT), Mahan, Kerman, Iran. He had joined with the Atomic and Molecular Group at the Queen's University of Belfast in 1992 on a sabbatical leave. He also spent two sabbatical leaves at eh Flinders University of South Australia, Adelaide, SA, Australia on 1997 and 2005. He joined with the Physics Department at the University of Western Ontario, London, Ontario, Canada in 2000 as a visiting professor. He published more than 50 peer-reviewed papers with over 500 citations. He published a textbook on the Experimental Methods in Atomic Physics. His topics of work are scattering problems in atomic, molecular and optical areas as well as optical communication and applications of fibers in industry and medicine. Prof. Bolorizadeh is a member of the Optical Society of America, a member of Iranian Physical Society and a member of the Optics and Photonics Society of Iran (OPSI). He also served as the Head of the Organizing Committee of OPSI for 3 years. He was also recognizes as the Veteran of OPSI in 2010.

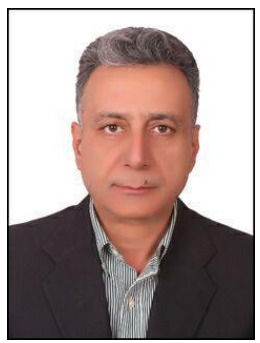

Ali Reza Ahmadi Ph.D. in Mechanical Engineering from University of Kansas. Born In Kerman, Iran. B.S. in CE from USIU, California, U.S.A. M.S. in CE from UCI, California, U.S.A. D.E. in $\mathrm{CE}$ from $\mathrm{KU}$ Kansas, U.S.A. Ph.D. in ME from KU Kansas, U.S.A. $\mathrm{He}$ is a Faculty member of the Mechanical Engineering Department at Kerman Graduate University of Technology. $\mathrm{He}$ has been active in using finite element technology modeling and simulation over 30 years. Dr Ahmadi has developed an open architecture finite element package to be used in developing finite element technology and solving governing equations for a variety of physical processes.

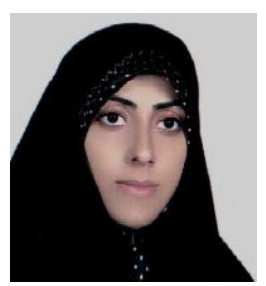

Motahare Sadat Hoseinian was born in Qom, Iran on September 11, 1984. She earned her B.Sc. in physics from A Zahra University, Tehran, Iran. Later, she earned M.Sc. in Graduate University of Advanced Technology (GUAT), Mahan, Kerman, Iran. She is currently a PhD student in atomic and molecular physics in GUAT. Her topic of work is, currently, optical sensors making use of nanoparticles in fibers to be applied in medicine and industry. She co-authored two books on the subjects of "Gold Nanoparticle", published by University of Tehran's Press (2016) and "Plasmonics, Fundamental and Application", published by Mana Books Publisher (2016). She published 20 papers in National Conferences and 4 peer-reviewed 
papers in nanotechnology, biotechnology and optical fiber sensors.

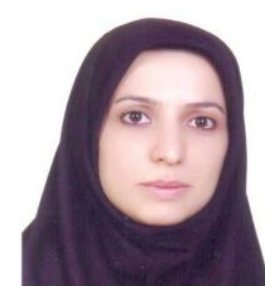

Maryam Alvanforoush earned her B.Sc. in physics from Yazd University, Yazd, Iran, and her M.Sc. in Photonics from Graduate University of Advanced Technology (GUAT), Mahan, Kerman, Iran. Her topic of work is Kerr Nonlinear Medium in Wagon Wheel Fiber.

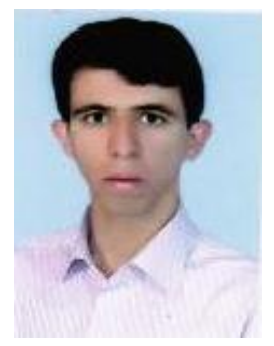

Ali Asghar Zakerifar is a MSc. Graduate from the Department of Photonics Engineering at Graduate University of Advanced Technology (GUAT). $\mathrm{He}$ has done his bachelor degree in Physics. His master's thesis in the field of Nonlinear Fiber Optics was on "Supercontinuum generation in wagon wheel fiber in abnormal dispersion". He published 5 papers in domestic Conferences and a paper in SPIE conference (2012). Regarding Nonlinear Fiber Optics and Supercontinuum Generation 